\title{
First Report of Nosocomial Outbreak of Vancomycin-Resistant Enterococcus Faecium Infection Among COVID-19 Patients Hospitalized in a Non-Intensive Care Unit Ward in Central Europe
}

\author{
Yashar Jalali ( $\nabla$ jalali_yashar@yahoo.com ) \\ 5th Department of Internal Medicine, University Hospital https://orcid.org/0000-0003-0991-1727 \\ Igor Šturdík \\ 5th department of internal medicine, University Hospital Bratislava, Ružinov \\ Monika Jalali \\ 5th department of internal medicine, University Hospital Bratislava, Ružinov \\ Ján Kyselovič \\ 5th department of internal medicine, University Hospital Bratislava, Ružinov \\ Adriána Liptáková \\ Comenius University in Bratislava Faculty of Medicine: Univerzita Komenskeho v Bratislave Lekarska fakulta \\ Andrea Kološová \\ Department of hospital hygiene and epidemiology, University Hospital in Bratislava, Ružinov \\ Miroslav Böhmer \\ Comenius University in Bratislava: Univerzita Komenskeho v Bratislave

\section{Tomáš Szemes} \\ Comenius University in Bratislava Faculty of Natural Sciences: Univerzita Komenskeho v Bratislave Prirodovedecka fakulta \\ Stanislav Stuchlík \\ Comenius University in Bratislava Faculty of Natural Sciences: Univerzita Komenskeho v Bratislave Prirodovedecka fakulta \\ Juraj Payer \\ 5th department of internal medicine, University Hospital Bratislava, Ružinov
}

\section{Research}

Keywords: Vancomycin-resistant Enterococcus faecium, antibiotic resistant, multidrug resistant, COVID-19, SARS-CoV-2, bacterial outbreak, healthcare-associated infection.

Posted Date: October 11th, 2021

DOI: https://doi.org/10.21203/rs.3.rs-955202/v1

License: (c) (i) This work is licensed under a Creative Commons Attribution 4.0 International License. Read Full License 


\section{Abstract}

Background: The COVID-19 pandemic in 2020 exerted immense pressure on health care systems worldwide, causing substantial resources to be diverted to respond to the pandemic. These changes raise the concern about the potential for reduction in adherence to long-established measures in the prevention of healthcare-associated infections (HAl). Enterococcus species account for most of human enterococcal HAl and multidrug-resistant infections and have become a major threat to modern public health. We examine the rise in the number of vancomycin resistant $E$. faecium blood stream and urinary tract infections in a COVID-19 department during an epidemiologic outbreak investigation to detect and eliminate nosocomial clusters of the bacteria.

Methods: Strain identification was performed by classical isolation and biochemical and cultivation methods. Antibiotic testing results were interpreted according to European committee on antimicrobial susceptibility testing (EUCAST) guidelines. Six isolated samples underwent whole genome sequencing (WGS) during the outbreak investigation. Isolate relatedness was determined using core genome multilocus sequence typing.

Results: WGS revealed two genotypically distinct VRE clusters, one of which had genetically closely related patient and environmental isolates. The cluster was terminated by enhanced infection control strategies.

Conclusions: This study provides the first description of an outbreak caused by vanA-ST17 E. faecium strains among COVID-19 patients in central Europe, and the first description of an outbreak caused by vanB-ST117 and vanA-ST17 E. faecium strains in Slovakia. This study can help raise awareness about the need for strict adherence to infection control measures and the implementation of rational antimicrobial stewardship as a routine part of COVID-19 management.

\section{Background}

Emergence of the COVID-19 pandemic during 2020 exerted immense pressure on health care systems worldwide (1). Diverting significant staff and financial resources and repurposing of hospital departments to face the critical lack of viable space for isolating the ever-rising number of infected patients to attempt to manage and overcome the pandemic, raised concerns among epidemiology and infection control communities about the potential for reduced adherence to long-established measures for the prevention of health associated infections ( $\mathrm{HAl}$ ). Although effects of such changes are uncertain $(2,3)$, current reports of increases in the occurrence of nosocomial bacterial infections within COVID-19 dedicated departments are alarming (2). However, the real extent of this issue is poorly known due to a lack of studies (3).

Although enterococcus species (namely E. faecalis and to a lesser extent E. faecium) are abundant as a commensal of the human intestine microbiome, in immunocompromised hosts, they can act as an opportunistic pathogen and cause lifethreatening infections such as sepsis, endocarditis, and urinary tract infections $(4,5)$. E. faecalis and E. faecium are the most common cause of human enterococcal health-associated and multidrug-resistant infections and due to their natural intrinsic resistance to several antimicrobials, including last resort antibiotics, and their capacity to acquire virulence, are recognized as major nosocomial pathogens (5). Since the first reported cases in the 1980s, vancomycin-resistant enterococcus (VRE) strains have become a major modern public health threat. In the European Union (EU), VRE populated-proportions of infection significantly increased in the past 5 years without geographic differences (6).

Surveillance and comparison of VRE infection cases before and during the pandemic (after repurposing of our Department of Internal Medicine into a Department for Critically ill patients with COVID-19), showed a significant rise in number of vancomycin resistant $E$. faecium blood stream and urinary tract infections. Here we describe an outbreak of VRE infection and its management in COVID-19 positive patients during January to April 2021.

\section{Methods}

\section{Clinical setting and infection control measures}

Page 2/11 
$5^{\text {th }}$ Department of Internal medicine of the University Hospital in Bratislava as the largest department in this filed-field in capital city of Slovakia, was repurposed for the treatment of critically ill, COVID-19 positive patients with internal disease co-morbidities, during the peak of second wave of COVID-19 pandemic (December 2020) in the country. From the beginning of December 2020 until the end of April 2021, 395 patients with COVID-19 were hospitalised in two separate isolation wards (ward A and ward B, with a capacity of 40 beds in total) and one isolation ICU (with a capacity of 8 beds) in the department. Enhanced infection control measures were applied for the isolation areas (isolation red zones), including use of personal protective equipment (PPE) with biosafety level 3 (BSL-3).

The general internal surveillance program of drug-resistant bacteria in the department flagged a considerable increase in number of positive VRE cases (all caused by E. faecium) in COVID-19 positive patients in December 2020 (a 60\% increase in comparison to the previous three months, (6 positive cases were diagnosed for period of three months between September to November 2020 versus 8 positive diagnosed cases during December 2020), as well as in comparison to the same period in the previous year cases (2 positive diagnosed cases during December 2019 in comparison to 8 positive diagnosed cases in December 2020). However, since most of hospitalized patients were transferred from other clinics, hospitals, or nursing homes, the positive VRE cases were considered possible imported cases rather than part of an in-department outbreak. After a change in antibiotic treatment and physical isolation, these patients were only followed until January 2021 and not included in this study.

In January 2021, we continued to record positive VRE and initiated the epidemiological investigation following the implementation of strategies for terminating the VRE outbreak. These strategies included:

1) implementing a new antibiotic therapy algorithm for treating bacterial super-infections and minimizing inappropriate initiation of antibiotic therapy (specifically, the use of cephalosporins). A combination of Augmentin and Clarithromycin was used for treating community-acquired pneumonia and Tazocin with an aminoglycoside was used for hospital-acquired pneumonia, until antibiotic susceptibility testing results were obtained. For positive cases of VRE, a combination of linezolid with doxycycline was used.

2) We implemented a point prevalence screening on all patients upon admission and once a week thereafter.

3) We intensified the frequency of surface disinfection (from once a day to twice a day every 12 hours) of all surface areas and used instruments in red and green zones (green zone are areas outside of red zones within the ward in which BSL 2 or lower was required). For disinfection, we used Incidin Oxyfoam S (Ecolab, Deutschland GmbH, Monheim am Rhein), Meliseptol Foam pure (B. Braun Melsungen AG, Germany) and Chloramine $0.2 \% / L$ solution.

4) Patients diagnosed with VRE were accommodated in same room (designated with a VRE positive sign), which had separated toilets or bedside toilets and higher hygienic precautions, and dedicated examination instruments. Complete isolation of VRE patients (one patient per room) was not possible during the peak of the pandemic. As VRE positive cases continued to emerge throughout February 2021 (although with a mild decrease in number; 7 cases in December 2020, 6 cases in January 2021, and 4 cases in February 2021), we performed environmental sampling for detection of VRE contaminations in red and green zones and all samples from diagnosed patients underwent further genetic examination for epidemiological mapping of transmission routes.

\section{Data and demographical information, inclusion, and exclusion criteria}

Data used in this study was gathered from all patients hospitalised in the red zone during a period of 4 months (beginning of January 2021 until the end of April 2021). All patients included in this study had a confirmed infection of SARS-CoV-2 based on a RT-PCR test. We recorded demographical data for each patient including age and gender, and collected information on the duration of hospitalisation, place of sampling, antimicrobial susceptibility, type of acquired infection (HAl, or non-healthcare associated infection, NHAI), whether the patient was dismissed or died during our monitoring, and the duration of hospitalisation before diagnosis of VRE infection. Other than positive COVID-19 PCR test and clinically relevant diagnosis of and VRE (positive samples from the urinary tract, haemoculture, decubitus, or sputum in patients with a high likelihood of bacterial infectious process such as elevated CRP and procalcitonin), no other inclusion or exclusion criteria were used in this study. 


\section{Environmental sampling, patient sampling, and testing methods}

In accordance with the internal guidelines of the department, sterile swab samples of the tonsils and nose, and samples of sputum and mid-stream urine, were collected for microbiological investigation from all patients upon admission. Other specific microbiological sampling (haemoculture, stool culture, etc.) were performed as deemed necessary by the patient's physician. After detection of the VRE outbreak and implementation of stricter hygienic measures, we performed anal swab sampling for all patients in red zones at the time of admission and once a week thereafter. Blood sampling was performed using peripheral intravenous puncture. Urine was collected by mid-stream urine sampling or via urinary catheter.

Environmental sampling was conducted twice during February 2020 in both green and red zones. Sampling was performed using sterile swab samples of surfaces and examination instruments We collected a total of 30 environmental samples ( $20 \mathrm{from}$ the red zone and 10 from the green zone).

Patient samples and environmental samples were processed by conventional methods approved by the Slovak Ministry of Health. Bacterial identification was performed by standard bacterial culture from biological specimens (urine, swabs, etc.) growth on solid media using Columbia blood agar with 7\% sheep blood (EnviroLab), and Uriselect 4 chromogenic agar (EnviroLab) for urine culture (Medirex a.s.). Strain identification was performed by matrix-associated laser desorption/ionization time-of-flight mass spectrometry (MALDI-TOF MS) (Bruker, Massachusetts, USA). Antimicrobial susceptibility testing was performed using the disc diffusion test on Mueller-Hinton agar culture and results were interpreted according to the EUCAST guideline (European Committee on Antimicrobial Susceptibility Testing).

\section{DNA extraction, library preparation and sequencing}

DNA was extracted from plated samples using the DNeasy Blood and Tissue Kit (Qiagen, Hilden, Germany) using a protocol for isolation of gram-positive bacteria following the manufacturer's instructions. DNA libraries were prepared using Nextera XT DNA Library Prep Kit (Illumina Inc., San Diego, CA, USA) as described in the protocol, followed by library validation using Qubit dsDNA high sensitivity assay (LifeTechnologies, Eugene, OR, USA) and Agilent ${ }^{\circledR}$ HS DNA Chip on Agilent ${ }^{\circledR}$ Technologies 2100 Bioanalyzer (Santa Clara, CA, USA). Finally, DNA libraries were normalized to a concentration of $4 \mathrm{nM}$, denatured, and sequenced on a MiSeq system (Illumina Inc., San Diego, CA, USA) using MiSeq Reagent kit v3 with paired end of 2x 300 bp reads.

\section{Data analysis}

Adapters and low-quality ends of sequenced reads were removed using Trimmomatic version 0.36 (7) based on quality control statistics generated by FastQC version v0.11.5 (8). After trimming, fragments without sufficient length of both reads (>35 bp) were removed from the data set. We used SPAdes version 3.10.1 (9) for de novo assembly of data and BLAST software (Basic Local Alignment Search Tool) version 2.10 .1 (10) for the classification of sequences. Computational analyses were written and executed using the SnakeLines framework version $0.11 .6(11,12)$. We used Type Genome Server (TYGS) version 281 (13) for genome-based taxonomy analysis and visualization (Figure 1). Sequences were mapped with Burrow-Wheeler Aligner (BWA) version 0.6 (14) against the van genes listed in Table 4. Then we used multilocus Sequence Typing (PubMLST) (15). Finally, the percentage of similarity between de novo assembled samples (Table 2) was determined with BLAST (10).

\section{Results}

We diagnosed a total of 10 positive VRE cases during January and February 2021 ( 6 cases and 4 cases, respectively). After implementing strict hygienic measures and targeted antibiotic therapy during these two months (described above), the number of new cases gradually decreased, and clusters were terminated. During March and April 2021, no new cases of VRE were reported.

Out of 10 diagnosed cases, 8 cases were isolated from male patients and 2 cases from female patients. The mean age of positive VRE diagnosed male patients was 73 that of female patients was 80 . All diagnosed cases of VRE were HAI. Diagnosed patients were hospitalized on average for 14 days before being diagnosed with VRE infection and were hospitalized on average for 21 days in total. Out of 10 cases, 3 were isolated from haemocultures and 7 were isolated from urine. All patients diagnosed 
with urinary tract VRE infection at the time of diagnosis had urinary catheters. Only one patient included in this study died during hospitalization. No samples were isolated from patients hospitalized in the ICU. None of the patients diagnosed with VRE were under pulmonary ventilation (by means of orotracheal intubation); however, all patients were under non-invasive humidified highflow oxygen therapy (Airvo 2, Ficher \& Paykel Healthcare Limited, New Zealand).

All isolated cases showed complete resistance to ampicillin, nitrofurantoin, and vancomycin. Resistance to aminoglycosides (gentamycin) was $80 \%$, and all samples were $100 \%$ susceptible to linezolid and tigecycline (Graph 1).

When controlled, all diagnosed patients were on dual antibiotic therapy for an extended period of time (at least 7 days) before being diagnosed with VRE. The majority of patients (7 out of 10) were on dual combination antibiotic therapy, including one $3^{\text {rd }}$ generation cephalosporin and one macrolide (azithromycin). Two patients were on dual combination antibiotic therapy including one $3^{\text {rd }}$ generation cephalosporin and metronidazole. This means that 9 out of 10 patients were on at least on one $3^{\text {rd }}$ generation cephalosporin before diagnosis (7 patients were on ceftriaxone, one patient was on cefotaxime and one patient was on ceftazidime). Only one patient received dual antibiotic therapy that did not include cephalosporins before diagnosis of VRE (piperacillin-tazobactam in combination with clindamycin) (Graph 2).

Four samples from patients and two environmental samples from the red zone taken in February 2021 were subjected to whole genome sequencing (WGS) (minimum, maximum, and mean coverage $=80.8,127.8,105.8$, respectively). The minimum, maximum, and mean N50 (the length of the sequence of the shortest contig making up 50\% of the total length of the genome together with the larger contigs) was 42691, 50654, and 46572, respectively. The minimum, maximum, and mean L50 (the smallest number of contigs whose sum of lengths is half the size of the genome) was 18,20 , and 20 , respectively.

Samples were numbered 1 to 6 (Table 1). Sample no. 1 and 4 were isolated from patients in ward A, sample no. 3 and 6 from patients in ward B, and sample no. 2 and 5 were isolated from the environment in ward A. No environmental samples from ward B were positive. All four patient samples were isolated from male patients. One sample (sample no. 1) was isolated from haemoculture, while the other three samples were isolated from urine (Table 1).

Table 1. Samples included in whole genome sequencing, their place of isolation, and van gene differences

\begin{tabular}{|c|c|c|c|c|c|c|}
\hline Isolated samples & No. 1 & No. 2 & No. 3 & No. 4 & No. 5 & No. 6 \\
\hline \multicolumn{7}{|l|}{ Place of sampling } \\
\hline Ward A & $x$ & $x$ & & $x$ & $x$ & \\
\hline Ward B & & & $x$ & & & $x$ \\
\hline Sample from patient & $x$ & & $x$ & $x$ & & $x$ \\
\hline Sample from environment & & $x$ & & & $x$ & \\
\hline Isolated from urine & & & $x$ & $x$ & & $x$ \\
\hline Isolated form haemoculture & $x$ & & & & & \\
\hline Van operons & vanA & $\operatorname{van} A$ & vanB & $\operatorname{van} A$ & $\operatorname{van} A$ & vanB \\
\hline
\end{tabular}

No.: number, van operon: operons related to vancomycin resistance in enterococci

The analysis of the results showed two clusters of closely related strains (Figure 1). Cluster 1 (C1), including sample no. 3 and sample no. 6, were almost identical when their genome was compared (99.9\% percent similarity) (Table 2). Cluster 2 (C2) comprised of sample numbers 1, 4, 2, and 5 (Figure 1). Sample no. 1 and 4 were virtually identical (Table 2). When compared to the known sequenced genomes, C1 samples were almost identical to known sequenced genomes of E. faecium LR135401.1 (99.98\% similarity) and isolated samples of C2 were similar to the known genome of E. faecium CP019970.1 (99.62\%) (Table 2). Both samples of C1 were identified as vanB-ST117, whereas all samples of C2 were identified as vanA-ST17 (Table 3). 
Table 2. Percentage of similarity between de novo assembled samples using BLAST (10).

\begin{tabular}{llllllllll} 
Similarity $\%$ & Sample & Sample & Sample & Sample & Sample & Sample & CP038996.1 & LR135419.1 & CP019970.1 \\
& 1 & 2 & 3 & 4 & 5 & 6 & & & \\
Sample 1 & $\mathrm{x}$ & 99,99 & 99,63 & 100 & 99,98 & 99,62 & 99,63 & 99,62 & 99,79 \\
\hline Sample 2 & 99,99 & $\mathrm{x}$ & 99,65 & 99,99 & 99,97 & 99,64 & 99,62 & 99,64 & 99,93 \\
\hline Sample 3 & 99,63 & 99,65 & $\mathrm{x}$ & 99,64 & 99,61 & 99,99 & 100 & 100 & 99,99 \\
\hline Sample 4 & 100 & 99,99 & 99,64 & $\mathrm{x}$ & 99,98 & 99,62 & 99,95 & 99,62 & 99,78 \\
\hline Sample 5 & 99,98 & 99,97 & 99,61 & 99,98 & $\mathrm{x}$ & 99,6 & 99,95 & 99,6 & 99,91 \\
\hline Sample 6 & 99,62 & 99,64 & 99,99 & 99,62 & 99,6 & $\mathrm{x}$ & 99,99 & 99,97 & 99,98 \\
\hline CP038996.1 & 99,63 & 99,62 & 100 & 99,95 & 99,95 & 99,99 & $\mathrm{x}$ & $\mathrm{x}$ & $\mathrm{x}$ \\
\hline LR135419.1 & 99,62 & 99,64 & 100 & 99,62 & 99,6 & 99,97 & $\mathrm{x}$ & $\mathrm{x}$ & $\mathrm{x}$ \\
\hline CP019970.1 & 99,79 & 99,93 & 99,99 & 99,78 & 99,91 & 99,98 & $\mathrm{x}$ & $\mathrm{x}$ & $\mathrm{x}$
\end{tabular}

CP038996.1, LR135419.1, CP019970.1: Known E. faecium reference genomes (16)

Table 3. Multilocus sequence typing, van operon genotypes mapped with BWA against van genes references

\begin{tabular}{|c|c|c|c|c|c|c|c|}
\hline & $\begin{array}{l}\text { Sample } \\
1\end{array}$ & $\begin{array}{l}\text { Sample } \\
2\end{array}$ & $\begin{array}{l}\text { Sample } \\
3\end{array}$ & $\begin{array}{l}\text { Sample } \\
4\end{array}$ & $\begin{array}{l}\text { Sample } \\
5\end{array}$ & $\begin{array}{l}\text { Sample } \\
6\end{array}$ & Genomic sequence \\
\hline vanA & $x$ & $x$ & & $x$ & $x$ & & $\begin{array}{l}\text { NZ_WQKY01000109.1:4393-5424 } \\
\text { Enterococcus faecium strain 14S_RHH008 } \\
\text { Contig_109, whole genome shotgun sequence }\end{array}$ \\
\hline $\operatorname{van} A$ & $\mathrm{x}$ & $\mathrm{x}$ & & $x$ & $x$ & & $\begin{array}{l}\text { NG_048325.1 Enterococcus faecium C864 } \\
\text { vanA gene for D-alanine-(R)-lactate ligase } \\
\text { vanA, complete CDS }\end{array}$ \\
\hline $\operatorname{vanB}$ & & & $x$ & & & $x$ & $\begin{array}{l}\text { NG_048333.1 Enterococcus faecium TSGH1 } \\
\text { vanB gene for D-alanine-(R)-lactate ligase } \\
\text { vanB, complete CDS }\end{array}$ \\
\hline vanB2 & & & $x$ & & & $x$ & $\begin{array}{l}\text { NG_048333.1:101-1129 Enterococcus } \\
\text { faecium TSGH1 vanB gene for D-alanine-(R)- } \\
\text { lactate ligase vanB, complete CDS }\end{array}$ \\
\hline ST & 17 & 17 & 117 & 17 & 17 & 117 & \\
\hline $\begin{array}{l}\text { Clonal } \\
\text { Complex }\end{array}$ & CC17 & CC17 & CC17 & CC17 & CC17 & CC17 & \\
\hline
\end{tabular}

ST: sequence type, CC: clonal complex

\section{Discussion}

At least two studies reported a similar rise in VRE cases in COVID-19 positive patients hospitalized in intensive care units in Germany and Italy $(3,17)$. However, it is still not clear if the increase in VRE cases is directly connected to SARS-CoV-2 infection and its complications, or whether it is related to a disruption in vigorous infection control measures, or both.

\section{Significance of antimicrobial stewardship}

The role of antimicrobial treatment in the development and epidemiology of VRE is well documented (18). In a meta-analysis published by Carmeli et al. (19), $3^{\text {rd }}$ generation cephalosporins and parenterally introduced metronidazole, "likely due to their activity against nonenterococcal aerobic enteric flora", have been shown to be highly significant independent risk factors for VRE 
infection (19). The same study reports that the risk of VRE increased regardless of the duration of therapy with these agents. Other studies have confirmed the relation between the use of extended spectrum cephalosporins and increased risk of VRE infection (18). These reports correspond with the results of our study. Nine out of 10 patients diagnosed with VRE were on one $3^{\text {rd }}$ generation cephalosporins, 2 of which were at the same time in combination therapy with parenteral metronidazole. Limiting cephalosporins administration with strict antibiotic targeted therapy and using dual antibiotic treatment (linezolid in combination with doxycycline) for VRE positive patients resulted in a significant drop and control of positive cases. Hence, understandably, the appropriate use of cephalosporins only for targeted treatment, and the proper choice for initiation of antibiotic treatments and choice of antibiotics are of immense importance (20).

\section{Significance of environmental hygiene}

Given that the surge in the number of VRE positive cases was initially reported when many patients were transferred from nursing homes, other wards, or other hospitals, it is possible that VRE species were import into our COVID-19 ward. However, the fact that new cases emerged in January and February (when point prevalence control for colonisation of VRE at time of patient admission was adopted) suggests a role of VRE transmission within the wards. Indirect surface contact has been described as a frequent route for VRE transmission in hospital settings (21). Clonal complex 17 (CC17) is a well-documented global polyclonal cluster of hospital adapted vancomycin-resistant Enterococcus faecium (5). So far, several sequence types (ST) have been described for their multidrug resistance ability and different levels of virulence $(5,22,23)$. ST17 and ST117 are among the most well-studied clones involved in in-hospital outbreaks and have been shown to express high levels of antimicrobial resistance and virulence $(4,5)$. In recent years, isolates belonging to sequence type 117 are increasingly identified from clinical isolates in many European health institutions $(4,5)$.

In our study, we identified two CC17 clones from ward A and B. The clone from ward A belongs to the vanA-ST17 lineage. A study by Si-Ho Kim et al. (2021) suggests that ST17 is a predictor of subsequent bacteraemia (22). One of the two samples from this clone was isolated from haemoculture, and the patient with positive haemoculture sample died during the study. Isolated clones from ward B belonged to the vanB-ST117 lineage; this clone is known to have caused nosocomial outbreaks in several European countries (24-26). Due to a lack of information, we could not evaluate the prevalence of VRE infections caused by these lineages in Slovakia. WGS analysis showed close genetic relations between environmental VRE samples and VRE samples isolated from patients, which points to the role of transmission through contaminated surfaces in ward A. Given that we implemented point prevalence control at the time of admission, which rules out colonisation with VRE, we believe that the lack of environmental isolation from ward B is most likely due to technical and human errors in sampling. Enhanced infection controls were effective in terminating transmission chains. The sharp increase in the number of hospitalized patients, incomplete adherence to infection control measures due to lack of medical personnel, massive workload pressure, and technical complications of proper isolation of patients are important and concerning parameters in the development of this outbreak and can potentially lead to similar situations in future.

Long hospitalization durations due to COVID-19 disease complications, as well as urinary catheterization, can be considered separate risk factors in the development of VRE infection in this study.

\section{Conclusions}

This study provides the first description of an outbreak caused by vanA-ST17 and VanB-ST117 E. faecium strains in Slovakia.

Importing VRE to COVID-19 wards by colonised patients from nursing homes or other clinics can be a real epidemiological and hygienic threat. To help prevent future VRE outbreaks, anal swab sampling at the time of patient admission (specially from nursing homes or transfers from other wards/hospitals) and in-ward temporary isolation until a negative result is confirmed could be considered.

Extensive and/or inappropriate use of antimicrobials (especially extended spectrum cephalosporins) and insufficient adherence to infection control measures in immunocompromised/immune dysregulated COVID-19 patients due to lack of medical personnel, massive workload pressure, and technical complications seem to be the likely drivers of current VRE outbreak. There 
is a need to raise awareness about the importance of adhering to infection control measures along with the implementation of rational antimicrobial stewardship to reduce morbidity and mortality related to healthcare-associated infections as a routine part of COVID-19 management.

\section{Abbreviations}

AMP: ampicillin, AZM: azithromycin, BLAST: Basic Local Alignment Search Tool, BWA: Burrow-Wheeler Aligner, CAZ: ceftazidime, CC17: Clonal Complex 17, CLI: clindamycin, COVID-19: Coronavirus Disease 2019, CRO: ceftriaxone, CTX: cefotaxime, EU: European Union, EUCAST: European Committee on Antimicrobial Susceptibility Testing, GEN: gentamycin, HAl: Health Associated Infection, LZD: linezolid, MALDI-TOF MS: Matrix-Associated Laser Desorption/lonization Time-of-Flight Mass Spectrometry, MLST: Multilocus Sequence Typing, MTZ: metronidazole, MDR: Multidrug Resistant, NIT: nitrofurantoin, SARSCoV-2: Severe Acute, Respiratory Syndrome Coronavirus 2, RT-PCR: Reverse Transcriptase Polymerase Chain Reaction, ST17: Sequence Type 17, ST117: Sequence Type 117, TGC: tigecycline, TYGS: Type Genome Server, TZP: piperacillin-tazobactam, VAN: vancomycin, VRE: Vancomycin Resistant Enterococcus spp., WGS: Whole Genome Sequencing

\section{Declarations}

Ethics and approval and consent to participate: In accordance with local legislation and institutional requirements, ethical approval for this study and informed consent by participants was not required.

Consent for publication: Not applicable

Availability of data and materials: The datasets used and/or analysed during the current study are available from the corresponding author on reasonable request

Competing interest: There is no conflict of interest for any of authors

Funding: Not applicable

Authors' contributions: Yashar Jalali 50\%, Igor Šturdík: 5\%, Monika Jalali: 5\%, Ján Kyselovič: 5\%, Adriána Liptáková 5\%, Andrea Kološová 5\%, Miroslav Böhmer 10\%, Tomáš Szemes 5\%, Stanislav Stuchlík 5\%, Juraj Payer 5\%, all authors read and approved the final manuscript.

\section{References}

1. Verelst F, Kuylen E, Beutels P. Indications for healthcare surge capacity in European countries facing an exponential increase in coronavirus disease (COVID-19) cases, March 2020. Euro Surveill. 2020;25(13).

2. Rawson TM, Moore LSP, Castro-Sanchez E, Charani E, Davies F, Satta G, et al. COVID-19 and the potential long-term impact on antimicrobial resistance. J Antimicrob Chemother. 2020;75(7):1681-4.

3. Kampmeier S, Tönnies H, Correa-Martinez CL, Mellmann A, Schwierzeck V. A nosocomial cluster of vancomycin resistant enterococci among COVID-19 patients in an intensive care unit. Antimicrobial Resistance \& Infection Control. 2020;9(1):154.

4. Lee T, Pang S, Abraham S, Coombs GW. Antimicrobial-resistant CC17 Enterococcus faecium: The past, the present and the future. J Glob Antimicrob Resist. 2019;16:36-47.

5. Baptiste MOAaKE. Vancomycin-Resistant Enterococci: A Review of Antimicrobial Resistance Mechanisms and Perspectives of Human and Animal Health. Microbial Drug Resistance. 2018;24(5):590-606.

6. (EARS-Net) ECfDPaCAritEE. Annual Epidemiological Report. Stockholm; 2020.

7. Bolger AM, Lohse M, Usadel B. Trimmomatic: a flexible trimmer for Illumina sequence data. Bioinformatics. 2014;30(15):2114-20.

8. Bioinformatics B. FastQC: a quality control tool for high throughput sequence data. In: Bioinformatics B, editor.: Babraham Bioinformatics; 2010. 
9. Bankevich A, Nurk S, Antipov D, Gurevich AA, Dvorkin M, Kulikov AS, et al. SPAdes: a new genome assembly algorithm and its applications to single-cell sequencing. J Comput Biol. 2012;19(5):455-77.

10. Altschul SF, Gish W, Miller W, Myers EW, Lipman DJ. Basic local alignment search tool. J Mol Biol. 1990;215(3):403-10.

11. Budis J, Krampl W, Kucharik M, Hekel R, Goga A, Lichvar M, et al. SnakeLines: integrated set of computational pipelines for sequencing reads2021 June 01, 2021:[arXiv:2106.13649 p.]. Available from:

https://ui.adsabs.harvard.edu/abs/2021arXiv210613649B.

12. Köster J RSS. A scalable bioinformatics workflow engine. Bioinformatics2012.

13. Meier-Kolthoff JP, Göker M. TYGS is an automated high-throughput platform for state-of-the-art genome-based taxonomy. Nature Communications. 2019;10(1):2182.

14. Li H, Durbin R. Fast and accurate long-read alignment with Burrows-Wheeler transform. Bioinformatics. 2010;26(5):589-95.

15. Jolley KA, Bray JE, Maiden MCJ. Open-access bacterial population genomics: BIGSdb software, the PubMLST.org website and their applications. Wellcome Open Res. 2018;3:124.

16. Van der Auwera GA, Carneiro MO, Hartl C, Poplin R, Del Angel G, Levy-Moonshine A, et al. From FastQ data to high confidence variant calls: the Genome Analysis Toolkit best practices pipeline. Curr Protoc Bioinformatics. 2013;43:11 0 1- 0 33.

17. Cataldo MA, Tetaj N, Selleri M, Marchioni L, Capone A, Caraffa E, et al. Incidence of bacterial and fungal bloodstream infections in COVID-19 patients in intensive care: An alarming "collateral effect". J Glob Antimicrob Resist. 2020;23:290-1.

18. Kritsotakis El, Christidou A, Roumbelaki M, Tselentis Y, Gikas A. The dynamic relationship between antibiotic use and the incidence of vancomycin-resistant Enterococcus: time-series modelling of 7-year surveillance data in a tertiary-care hospital. Clin Microbiol Infect. 2008;14(8):747-54.

19. Carmeli Y, Eliopoulos GM, Samore MH. Antecedent treatment with different antibiotic agents as a risk factor for vancomycinresistant Enterococcus. Emerg Infect Dis. 2002;8(8):802-7.

20. Yim J, Smith JR, Rybak MJ. Role of Combination Antimicrobial Therapy for Vancomycin-Resistant Enterococcus faecium Infections: Review of the Current Evidence. Pharmacotherapy. 2017;37(5):579-92.

21. Wagenvoort JH, De Brauwer El, Penders RJ, Willems RJ, Top J, Bonten MJ. Environmental survival of vancomycin-resistant Enterococcus faecium. J Hosp Infect. 2011;77(3):282-3.

22. Kim S-H, Cho SY, Kim HM, Huh K, Kang C-I, Peck KR, et al. Sequence type 17 is a predictor of subsequent bacteremia in vancomycin-resistant Enterococcus faecium-colonized patients: a retrospective cohort study. Antimicrobial Resistance \& Infection Control. 2021;10(1):108.

23. Fang H, Froding I, Ullberg M, Giske CG. Genomic analysis revealed distinct transmission clusters of vancomycin-resistant Enterococcus faecium ST80 in Stockholm, Sweden. J Hosp Infect. 2021;107:12-5.

24. Weber A, Maechler F, Schwab F, Gastmeier P, Kola A. Increase of vancomycin-resistant Enterococcus faecium strain type ST117 CT71 at Charité - Universitätsmedizin Berlin, 2008 to 2018. Antimicrobial Resistance \& Infection Control. 2020;9(1):109.

25. Kerschner H, Cabal A, Hartl R, Machherndl-Spandl S, Allerberger F, Ruppitsch W, et al. Hospital outbreak caused by linezolid resistant Enterococcus faecium in Upper Austria. Antimicrobial Resistance \& Infection Control. 2019;8(1):150.

26. Abdelbary MHH, Senn L, Greub G, Chaillou G, Moulin E, Blanc DS. Whole-genome sequencing revealed independent emergence of vancomycin-resistant Enterococcus faecium causing sequential outbreaks over 3 years in a tertiary care hospital. European Journal of Clinical Microbiology \& Infectious Diseases. 2019;38(6):1163-70.

\section{Figures}




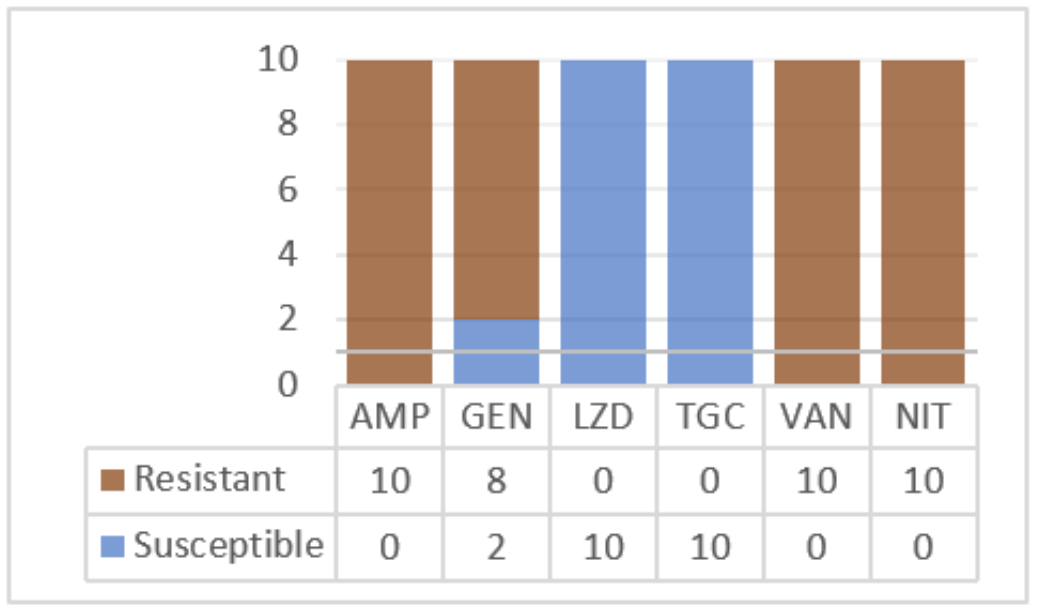

\section{Figure 1}

Graph 1. Antibiotic resistance profile of isolated cases of E. faecium AMP: ampicillin, AZM: azithromycin, CAZ: ceftazidime, CLI: clindamycin, CRO: ceftriaxone, CTX: cefotaxime, GEN: gentamycin, LZD: linezolid, MTZ: metronidazole, NIT: nitrofurantoin, TGC: tigecycline: VAN: vancomycin, NIT: nitrofurantoin, TZP: piperacillin-tazobactam.

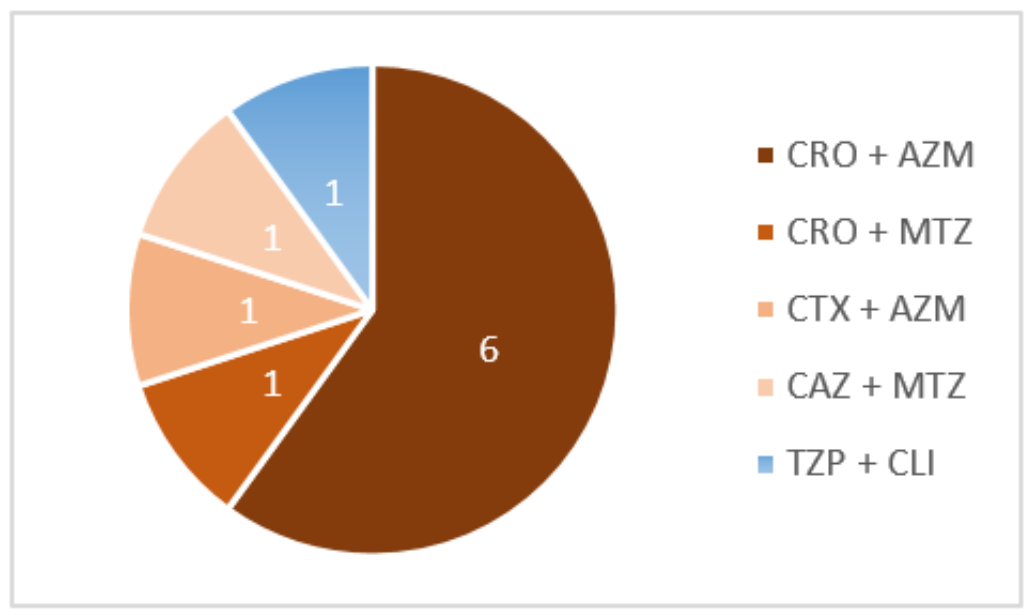

\section{Figure 2}

Graph 2. Number of diagnosed cases in relation to antibiotic combination treatment AMP: ampicillin, AZM: azithromycin, CAZ: ceftazidime, CLI: clindamycin, CRO: ceftriaxone, CTX: cefotaxime, GEN: gentamycin, LZD: linezolid, MTZ: metronidazole, NIT: nitrofurantoin, TGC: tigecycline: VAN: vancomycin, NIT: nitrofurantoin, TZP: piperacillin-tazobactam. 


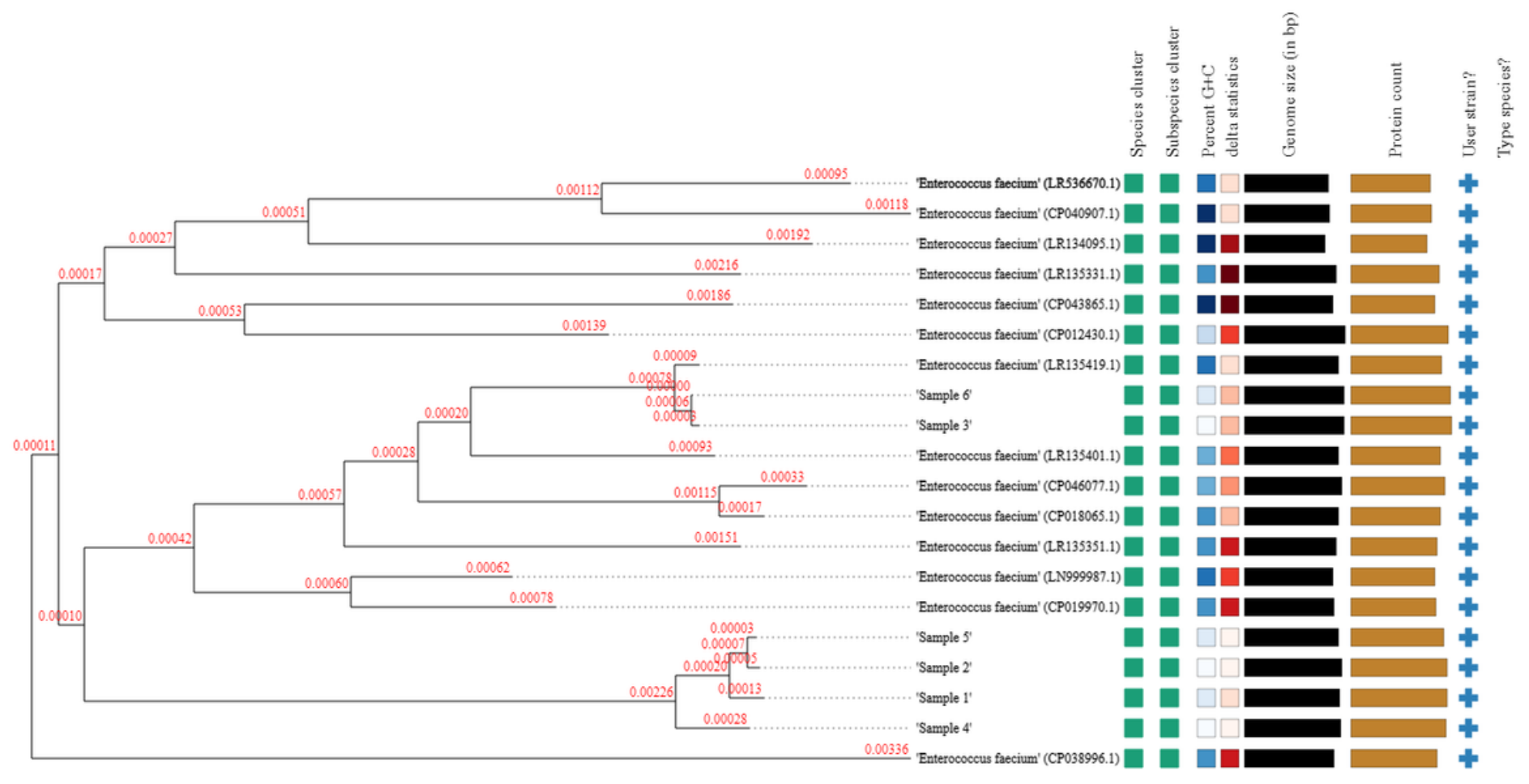

Figure 3

Phylogenetic tree (whole-genome sequence-based) of de novo assembled samples using TYGS (13). 